\title{
Sistemas complejos adaptativos y simulación computacional en Arqueología*
}

\author{
Complex adaptative systems and computational simulation in Archaeology
}

\author{
Salvador Pardo-Gordóa
}

\section{RESUMEN}

Tradicionalmente el concepto "complejidad" se utiliza como sinónimo de "sociedad compleja" para referirse a grupos humanos que presentan determinadas características tales como urbanismo, desigualdad y jerarquización. Con la introducción de los Sistemas no lineales y de los Sistemas Complejos a la disciplina arqueológica dicho concepto está siendo matizado. Este viraje teórico ha supuesto el auge de las simulaciones computacionales como método de análisis de procesos históricos. Este trabajo tiene un doble objetivo: presentar la corriente teórica caracterizada por el pensamiento generativo que se está llevando a cabo en la disciplina arqueológica e ilustrar una aplicación concreta de la simulación computacional basada en agentes a un problema arqueológico: la dispersión de las primeras producciones cerámicas en el Mediterráneo occidental. the dispersal of the first ceramic production in the western Mediterranean.

Palabras clave: Teoría y metodología arqueológica; Sistema Complejo Adaptativo; Simulaciones computacionales; Modelos basados en agentes; Expansión del Neolítico; Evolución cultural; Mediterráneo occidental.

Key words: Archaeological Methods and Theory; Complex Adaptive Systems; Computational Modelling; Agentbased; Neolithic Spread; Cultural evolution; Western Mediterranean.

\section{INTRODUCCIÓN}

La disciplina arqueológica desde las tres últimas décadas está desarrollando una praxis donde la visión inductiva del pasado da paso a la experimentación sistemática y la validación de hipótesis, conocidas como visión generativa (Epstein y Axtell 1997; Epstein 1999; Kohler 2000). Esta praxis se centra en la generación de escenarios para observar cuál de ellos se aproxima más al registro arqueológico. Introduce conceptos y métodos propios de la teoría de los sistemas no lineales y la teoría de la complejidad que permiten investigar de manera fiable diferentes procesos arqueológicos haciendo hincapié en cuestiones ausentes del registro ing in archaeology and to present a concrete applicati of agent-based modelling to an archaeological problem.

* Este artículo se ha elaborado gracias a la beca predoctoral BES2010-030715 financiada por el Ministerio de Economía y Competitividad (Gobierno de España) y los proyectos de investigación HAR2009-4360-C03-01, HAR2012-33111 y HAR2015-68962.

a Departament de Prehistòria, Arqueologia i Història Antiga. Universitat de València. Av/ Blasco Ibañez 28. 46010 València. Correo e.: salvador.pardo@uv.es orcid.org/0000-0002-1060-1526

Recibido 27-VI-2016; aceptado 13-IX-2016.

Copyright: (C) 2017 CSIC. Este es un artículo de acceso abierto distribuido bajo los términos de una licencia de uso y distribución Creative Commons Attribution (CC-by) España 3.0. 
arqueológico (Barton 2013) como la transmisión de la información.

En la comunidad arqueológica el concepto complejidad es muy atractivo y ha sido un tema de interés al menos desde el siglo XIX. Desde entonces se han elaborado numerosas aproximaciones desde diferentes perspectivas teóricas (Lull 1983; Johnson y Earle 2000; Chapman 2003; Yoffee 2005; Gilman 2013). La independencia en los detalles es, a veces notoria, pero el concepto suele ser sinónimo de sociedades complejas, entendidas como las propias de grupos humanos que exhiben desigualdades, urbanismo y una política jerarquizada entre otros elementos (Kohler 2012; Barton 2014). En las últimas décadas la concepción de "más complejo" se ha puesto en tela de juicio debido a la consideración de las sociedades humanas como sistemas abiertos, sin equilibrio y donde la materia y/o energía puede fluir (Allen 1997: 42). Esto significa que los conceptos "complejidad" y "sociedad compleja" no son sinónimos ya que cualquier sociedad humana, por muy simple que sea, debe ser considerada como un sistema complejo. Este enfoque de la complejidad permite que los componentes del sistema puedan cambiar sus especificaciones y evolucionar a lo largo del tiempo; este fenómeno se conoce como Sistema Complejo Adaptativo, $C A S$ en nomenclatura anglosajona ${ }^{1}$. Helbing (2010: 325) lo denomina enfoque fisicalista y consiste en la transferencia de propiedades de muchos sistemas de partículas a los sistemas sociales. La visión no ha estado exenta de críticas (Khalil 1995; Helmreich 1998, 1999).

El objetivo de este trabajo es contribuir al debate epistemológico en torno al análisis del registro arqueológico como $C A S$. Para ello se realiza un recorrido historiográfico en el uso de la modelización in silico ${ }^{2}$ y se describen las principales técnicas para el estudio de los Sistemas Complejos discerniendo cuál es la más útil para investigar los procesos arqueológicos y/o históricos. Finalmente, se expone un ejemplo concreto de modelización computacional basada en agentes: la expansión del Neolítico en el Mediterráneo occidental. La modelización está diseñada

\footnotetext{
${ }^{1}$ Este trabajo utiliza dicha nomenclatura, Complex Adaptive Systems, por su generalización en la comunidad científica y la propia familiarización del autor con ella.

${ }^{2}$ Hecho por ordenador.
}

para analizar la diversidad cultural a partir de un trasfondo teórico tomado prestado de las ciencias biológicas.

\section{SISTEMAS COMPLEJOS ADAPTATIVOS}

Los autores presentan diferentes definiciones de los CAS (p. ej. Holland 1995; Miller y Page 2007; Mitchell 2009) pero, en general, comparten su consideración como una clase específica de sistema abierto. A su vez el significado del concepto de Sistema Complejo puede variar según el investigador (Gell-Mann 1999: 17). En este trabajo se sigue a Mitchell (2009: 13) que lo define $^{3}$ como "un sistema en el cuál grandes redes de componentes sin control central y con reglas simples de operación origina un comportamiento colectivo complejo, un procesamiento sofisticado de información y una adaptación mediante aprendizaje o evolución”. En esta definición pueden rastrearse algunas de sus principales características como el carácter adaptativo y el procesado de la información. Algunas son muy interesantes cuando se aplican a las ciencias sociales y, por extensión, al ámbito arqueológico.

Los CAS son 'sistemas abiertos' que carecen de equilibrio debido a que requieren un flujo de energía para mantener el sistema (Allen 1997: 42; Cilliers 1998: 4). Por ejemplo, el ser humano para mantener su sistema (vida) captura energía mediante la ingestión de alimento y la respiración. Los Sistemas Complejos Adaptativos están formados por múltiples 'componentes' o agentes que se agregan y disgregan en una jerarquía encajada en la que los componentes inferiores se van agrupando con subsistemas superiores. Cuanto más complejo es un sistema más niveles organizativos presenta. Los $C A S$ pueden definirse también como redes estructuradas (complex networks, Barabási 2005, 2012). En términos sociales, estos componentes pueden considerarse como jerarquías de organización, ya sean familias nucleares dentro de un clan o individuos en un gremio de artesanos, por ejemplo. Esta clase de organización se asocia al crecimiento del sistema: componentes de más bajo nivel se agrupan en subgrupos y estos en un

\footnotetext{
${ }^{3}$ Traducción propia del autor.
}

Trab. Prehist., 74, N. ${ }^{\circ}$ 1, enero-junio 2017, pp. 9-25, ISSN: 0082-5638

doi: $10.3989 /$ tp.2017.12181 
nivel superior. En definitiva, los componentes del sistema tienden a la 'autoorganización' sin imposiciones internas o externas donde las interacciones entre los componentes de cada subgrupo tienden a ser más frecuentes y robustas que las interacciones con los componentes de un subgrupo diferente. Por ejemplo, esta característica es rastreable en el sistema fiscal del Imperio romano, donde los gobernadores provinciales y los legati ad census accipendos recaudaban los impuestos (Fernández Uriel 1995: 163) sin mediar una relación directa entre cada miembro del Imperio y el emperador. Una consecuencia directa del carácter organizativo de los $C A S$ es que el remplazo de un componente por otro tiene, por norma, poco impacto en el sistema. En el Imperio romano la muerte de un emperador y su sustitución por otro tenía muy poca influencia en el funcionamiento general del sistema fiscal imperial.

Otra característica de los Sistemas Complejos Adaptativos es su 'descomposición cercana' que, básicamente, significa que tienden a fragmentarse en sentido inverso a como se formaron; los grupos de más alto nivel se convierten en sistemas independientes desvinculándose de otros grupos $\mathrm{y}$, estos a su vez, pueden desglosarse en subgrupos (Barton 2014). El Imperio romano sigue siendo ilustrativo. Durante su máxima expansión abarcaba desde la península ibérica al Oriente Próximo y cuando diferentes fluctuaciones (crisis del siglo III: invasiones pueblos exteriores, crisis económica, política y social) alcanzaron una magnitud que el sistema no pudo absorber, el Imperio se descompuso en dos sistemas independientes al Occidente y al Oriente. A si mismo el Imperio romano de Occidente, antes de entrar en colapso en el siglo IV se descompuso en sus provincias administrativas. Un ejemplo similar se ha documentado en otros imperios como el chino (Johnson 1982).

La 'adaptabilidad' es una de las característica más importante de los $C A S$ ya que, como remarca Holland (1995), en si misma implica complejidad. La adaptabilidad y el 'procesado de información' consisten en la capacidad de transmitir y responder al entorno y/o a las interacciones con otros componentes del sistema. El procesado de información permite que los componentes del sistema identifiquen regularidades, schema según Gell-Mann (1994) o internal model según Holland (1995), y se anticipen a las consecuencias de sus acciones si el patrón es conocido o, lo que es lo mismo, adaptarse/evolucionar a partir del aprendizaje. Esta adaptación de los agentes del sistema (p. ej. células en un organismo, hormigas en una colonia o individuos en una sociedad) se basa en la información a escala local. Según Cilliers (1998: 3-4) las interacciones son dinámicas y complejas pero no determinan el comportamiento del sistema. Esta afirmación es muy discutible ya que pequeños cambios pueden iniciar cambios en cascada con un gran impacto en el sistema. Por ejemplo: una región dedicada a la agricultura sufre un periodo de sequía extrema y sus componentes (población) deciden aumentar la superficie de cultivo a partir de la tala sistemática de la masa forestal, dicha adaptabilidad puede ser contraproducente si provoca mayor aridez y alteración del régimen de precipitaciones. En definitiva, los agentes para sobrevivir deben ajustarse de forma constante a las condiciones del sistema como el corazón se adapta a las del sistema (cuerpo humano) mediante el incremento o la disminución de la frecuencia de contracciones rítmicas.

Otro rasgo clave de los $C A S$ es su condición 'no lineal', es decir, la dirección y escala de las modificaciones no son directamente proporcionales al proceso/procesos que las causan; pequeños cambios pueden producir grandes transformaciones y viceversa. Esta sensibilidad a las condiciones iniciales, conocida coloquialmente como efecto mariposa ${ }^{4}$ (Lorenz 1995), es una presuposición de la ciencia de la complejidad (Cilliers 1998: 4) e implica que los $C A S$ se mueven entre el orden y el caos, es decir, están lejos del equilibrio (Prigogine 1987: 99).

La ausencia de linealidad hace realmente complicada la predicción de los cambios del sistema a partir de las propiedades de sus componentes y se relaciona con la última propiedad de los sistemas complejos: la 'emergencia'. Esta corresponde a comportamientos no observados antes en el sistema (Waldrop 1992: 291) o, lo que es lo mismo, a estados visibles en el sistema pero no en sus componentes. Por ejemplo, la ola mejicana que se observa en numerosos eventos deportivos puede verse como una propiedad emergente de un sis-

\footnotetext{
${ }^{4}$ Concepto dado a conocer por Lorenz el 29-XII-1972 en su charla Predictability: does the flap of a butterfly's wings in Brazil set off a tornado in Texas? en el 139 meeting of American association for the advancement of science.
} 
tema formado por espectadores y sus decisiones (ovacionar o no) (Farkas et al. 2003; Miller y Page 2004). La emergencia a priori parece no ser problemática para su aplicabilidad en arqueología dado que intuitivamente la asociamos al surgimiento de una nueva tecnología (Kohler 2012).

Finalmente destacaremos el carácter 'equifinal' 5 de los Sistemas Complejos. Consiste en que los sistemas sin equilibrio pueden llegar a presentar idénticos resultados partiendo de condiciones iniciales diferentes, del mismo modo que las mismas causas pueden producir diferentes estados (Premo 2010). Por ejemplo, ¿cómo una empresa que se dedica a la venta de determinado producto ha conseguido incrementar los beneficios? La 'equifinalidad' implica que pudiera deberse a condiciones iniciales diferentes como la reducción de los costes de producción y el aumento de las ventas.

Las características propias de los CAS han influido en su uso a ciencias como la Física, Aeronáutica, Economía, Química, Sociología y Ciencias Deporte entre otras (véase Miller y Page 2007; Mitchell 2009).

Los $C A S$ pueden ser aplicados a la disciplina arqueológica al permitir comprender las dinámicas de las sociedades humanas y la consecuencia de las interacciones entre las mismas y/o con el mundo físico (Bentley 2003: 9). Es decir, el registro arqueológico, por su naturaleza, debe entenderse como el resultado de la propiedad emergente y nunca como la emergencia en si misma (Shennan 2002; Barton et al. 2012; Barton 2014). Esta visión teórico-metodológica aplicada a los procesos históricos ofrece un potencial de nuevos conocimientos que merece una mayor exploración y elaboración y supone un nuevo reto (Bernabeu et al. 2013; Bernabeu 2016).

\section{LA SIMULACIÓN COMPUTACIONAL: MODELANDO PROCESOS ARQUEOLÓGICOS IN SILICO}

El análisis e interpretación del registro arqueológico desde la perspectiva de los Sistemas Complejos Adaptativos requiere la elaboración

\footnotetext{
${ }^{5}$ La RAE no contempla el sustantivo equifinalidad ni su adjetivo, por lo que se ha utilizado la traducción de la nomenclatura inglesa.
}

de una teoría robusta en torno a las dinámicas evolutivas del sistema, así como la aplicación del enfoque generativo. Ello implica la utilización de modelos computacionales (modelos formales) que son representaciones simplificadas de la realidad estudiada. Con independencia del tipo de modelo elegido (p. ej. ecuaciones estructurales, análisis factorial o modelos basados en agentes), su aceptación depende de que cumpla dos requisitos básicos (Izquierdo et al. 2008: 101): a) capturar la realidad estudiada facilitando el desarrollo del proceso de inferencia que requiere la utilización del modelo formal y b) que sus resultados sean extrapolables a diversas situaciones. En definitiva, la simulación computacional se utiliza para representar algún aspecto concreto del mundo (GarcíaValdecasas 2011: 93). Implica la generación de múltiples agentes incluidos en un conjunto de reglas algorítmicas que actúan en base al procesamiento de la información (Barton 2014).

\subsection{La aplicación de la simulación computacional: un breve recorrido historiográfico}

Las aplicaciones informáticas en la investigación arqueológica grosso modo se introducen en torno a la década de 1950. La obra de James Doran (1970: 296-298) es la primera centrada en la simulación per se. Contiene un breve ensayo sobre la cibernética y su aplicación como herramienta útil para generar explicaciones del registro arqueológico. A partir de este momento y de forma sistemática se han elaborado síntesis en torno al estado de la cuestión del binomio arqueologíasimulación (Doran y Hodson 1975; Bell 1987; Mithen 1994; 2001; McGlade 2005; Costopoulos 2010; Lake 2013). Estas compilaciones rastrean la historia de la aplicación de la simulación en arqueología. Las revisiones historiográficas más recientes subdividen el uso de la modelización en cuatro etapas, cuya denominación se asocia a la popularidad de su aplicación en la investigación arqueológica (p. ej. Lake 2013). En otras disciplinas la nomenclatura recurre al ciclo vital: infancia, juventud, adolescencia y madurez (Sinclair y Seligman 1996). En los trabajos se concluye, sea cual fuere la terminología utilizada en la periodización, que en la actualidad la simulación

Trab. Prehist., 74, N. ${ }^{\circ}$ 1, enero-junio 2017, pp. 9-25, ISSN: 0082-5638

doi: $10.3989 /$ tp.2017.12181 
por ordenador ha adquirido una madurez metodológica al menos en algunos temas específicos (McGlade 2005: 555). Remarcan cómo el pesimismo existente en las primeras obras de síntesis sobre el futuro de las técnicas de simulación no se ha cumplido (Lake 2013).

La fase pionera de la simulación computacional corresponde a finales de los 1960 principios de los 1980. La formulación de la Nueva Arqueología (Binford 1962) introdujo el método hipotético-deductivo en el ámbito arqueológico mediante la aplicación del razonamiento matemático. Al mismo tiempo conceptos asociados a la Teoría de Sistemas (von Bertalanffy 1951) como los de auto-regulación (homeostasis), retroalimentación positiva o negativa, oscilación y equilibrio dinámico empiezan a tener un peso importante en el lenguaje arqueológico por su potencial (Doran 1970: 290). En este contexto sistémico y bajo "la sombrilla cibernética” aparecen las primeras modelizaciones computacionales, centradas en demostrar la utilidad de los métodos de simulación, reflejando el positivismo no dogmático del procesualismo. En esta primera fase, conocida como la edad de oro de la simulación (Costopoulos 2010: 23), hay una rápida diversificación y especialización de las temáticas tratadas mediante el método de la modelización y, salvo contadas excepciones (Wobst 1974; Wright y Zeder 1977), los trabajos se concentraron en replicar procesos agregados a nivel de sociedades (p. ej. Ammerman y CavalliSforza 1971, 1973,1979; Cooke y Renfrew 1979).

En la década de los 1980 hay una fase de aletargamiento debida al distanciamiento de la Arqueología de cualquier paradigma científico (McGlade 2005: 572). En ella el pensamiento postprocesualista, caracterizado por una gran diversidad de enfoques teóricos y planteamientos, influyó en el rechazo al razonamiento sistémico y en el abandono de las simulaciones computacionales (Costopoulos et al. 2010: 2).

La década de 1990 debe ser considerada la del diseño de los métodos en arqueología (Aldenderfer 1998: 94), entre los que resurge la simulación computacional (Lake 2001; McGlade 2005; Costopoulos y Lake 2010; Lake 2013). Esta emergencia de la modelización está en relación con la adopción en Arqueología de visiones teóricas provenientes de la Física como la Teoría de la Complejidad y los Sistemas no Lineales (Feigenbaum 1980; Waldrop 1992). En este contexto aparecen dos obras fundamentales donde se observa la dicotomía en la simulación computacional. Mithen (1990: 257) en la suya se manifiesta a favor de analizar las interacciones locales y la toma de decisiones de los agentes remarcando que las explicaciones globales (modelos de procesos agregados) son útiles cuando se entienden las preferencias individuales. En cambio van der Leeuw y McGlade (1997: 17), desde el posicionamiento contrario, argumentan que los sistemas dinámicos tienen una gran capacidad para contribuir al conocimiento de las sociedades estructuradas si sus procesos sociales se analizan como fenómenos evolutivos no lineales. Con independencia de la perspectiva utilizada ambas publicaciones ayudaron a discutir el lugar y el rol de la modelización en arqueología. No obstante, exceptuando algunos trabajos (Kohler 1993; Doran et al. 1994), en esta fase se generalizan los modelos basados en ecuaciones diferenciales aplicados a la dispersión de grupos humanos, siendo central la del Neolítico (p. ej. Cohen 1992; Aoki et al. 1996; Fort y Méndez 1999a, 1999b).

A partir de los 2000 la consolidación de la Teoría de la Complejidad, la aplicación de los Sistemas Complejos Adaptativos (p. ej. Bentley y Maschner 2003, 2008) y la obra de Kohler y Gumerman (2000) inician la segunda edad de oro de la simulación. Es el primer compendio de trabajos con aplicación de los $C A S$, marca la mayoría de edad de los modelos basados en agentes (McGlade 2005: 581) y es un manifiesto en pro de la reconciliación entre el procesualismo y el postprocesualismo (Kohler 2000: 14). Ahora los enfoques se diversifican. Van desde las modelizaciones dinámicas aplicadas a la dispersión de grupos humanos en diferentes contextos y cronologías a partir de la adición de variables a la fórmula de reacción-difusión de Fisher-SkellamKPP (detalles en Steele 2009) al estudio de cuestiones culturales utilizando como base el trabajo de Holland (1975) sobre el algoritmo genético (p. ej. Banks et al. 2013; Silva y Steele 2014). Paralelamente, se consolida la propuesta del Mithen (1990) sobre la necesidad de analizar las interacciones locales para comprender los fenómenos globales. Ello se debe en parte a la popularización de los Modelos Basados en Agentes, aplicados a numerosos problemas arqueológicos como la evolución del patrón de asentamiento, las dinámicas socioecológicas, la dispersión de grupos humanos 
y la evolución humana y/o cultural (Conolly et al. 2008; Barton et al. 2010; Kohler y Varien 2012; Crema et al. 2014a; Pardo Gordó et al. 2015).

Finalmente, otros enfoques empiezan a tener auge en la bibliografía arqueológica como el análisis de los procesos arqueológicos desde una perspectiva de redes complejas (Brughmans 2013; Coward 2013; Collar et al. 2015) y el análisis del registro arqueológico desde la modelización bayesiana (Ortman et al. 2007; Crema et al. 2014b; Edinborough et al. 2015; Rubio-Campillo 2016).

Esta breve revisión historiográfica permite observar la evolución en el uso de la simulación computacional por parte de la comunidad arqueológica, así como la diversidad de modelos formales aplicados. Además plantea la necesidad de discernir el tipo de modelo adecuado para analizar los fenómenos históricos y/o el registro arqueológico como un sistema complejo.

\section{2. ¿Qué clase de simulación computacional se debe utilizar en arqueología?}

Como se ha visto a lo largo del texto, el análisis de las sociedades humanas como sistemas complejos implica la utilización de los modelos computacionales, pero ¿qué método de simulación utilizar? La respuesta no es simple ya que existen diferentes aproximaciones, no excluyentes. Los modelos más utilizados en el ámbito de la arqueología son los dinámicos y los basados en agentes.

La modelización dinámica explica y predice el comportamiento evolutivo que exhibe el sistema a nivel macroscópico (Strogatz 1994: 2; Mitchell 2009: 15). Es muy útil para comprender la estructuración social y analizar los procesos sociales desde una perspectiva no-lineal (McGlade y van der Leeuw 1997: 19). La modelización dinámica puede ser descrita a partir de leyes físicas (p. ej. la segunda ley de la termodinámica) en lugar del procesamiento de la información, por lo que su lenguaje natural es una formulación matemática basada en ecuaciones diferenciales (Parunak et al. 1998; Quesada y Hassan 2012). Esta clase de modelos se conocen como aproximaciones de arriba-abajo (top-down en nomenclatura anglosajona) puesto que la modelización parte de la comprensión del todo para investigar las partes.
Dada su naturaleza top-down los componentes del sistema no están representados ni de forma explícita ni individual. Como las relaciones se modelizan a una escala superior es realmente complicado capturar las características individuales (Parunak et al. 1998: 20).

En los Modelos Basados en Agentes, en inglés los $A B M$ (sigla en inglés), o Modelos Basados en Individuos en el contexto de la Ecología, los individuos o agentes son entidades únicas y autónomas que difieren de otras entidades en el espacio y tiempo y usualmente interactúan con otras entidades o con el ambiente de manera local (Railsback y Grimm 2012: 4 y 10). La definición permite rastrear tres ideas fundamentales que los hacen realmente útiles a) el carácter único implica que cada agente, o grupo de ellos, es diferente de otros; b) el localismo de las interacciones, los agentes interactúan a partir del principio de vecindad y/o conectividad; c) el carácter autónomo de las entidades que forman los $A B M$ permite a los agentes disponer de un comportamiento libre e independiente según sus intereses, es decir, permite aproximarse al problema desde una perspectiva abajo-arriba (bottom-up en nomenclatura anglosajona).

Personalmente abogamos por la utilización de los $A B M$ cuando se analizan las sociedades humanas (y el registro arqueológico) como un sistema complejo puesto que es la metodología natural para capturar la propiedad emergente (Bonabeau 2002: 7280-7281) resultante de las interacciones entre las entidades a escala local. Además en el caso concreto de la Prehistoria estos modelos debido a su versatilidad en el momento de la lectura permiten examinar el comportamiento del sistema a partir de las interacciones locales, o bien, la influencia del sistema en el comportamiento de los individuos. En definitiva, los $A B M$ son una magnífica herramienta para investigar aquellos procesos que se caracterizan por presentar entidades con comportamiento.

\subsection{Simulando la hipótesis del autoestop cultural}

Tal y como se ha descrito anteriormente, a lo largo de las últimas décadas la Arqueología está adoptando una metodología que nos permite elaborar y testar hipótesis a partir de la obtención 
de resultados in silico. El problema clásico de la dispersión del Neolítico puede ayudarnos a comprender como se desarrolla dicho proceso.

La expansión del Neolítico en Europa es un tema recurrente en la bibliografía arqueológica, al menos desde que Childe (1925) planteara un origen exógeno. En la actualidad, la comunidad científica admite que la agricultura y ganadería se introducen en el Viejo Mundo a partir del Próximo Oriente. Este proceso de dispersión implicó una distribución espacio-temporal de los elementos del paquete neolítico (especies, tecnologías y prácticas sociales). En cambio subsiste un debate sobre los mecanismos de introducción de la agricultura en el viejo continente que puede articularse en dos posturas generales. La conocida como difusión démica pone el énfasis en el movimiento de la población y, por extensión, de las prácticas agrícolas. La segunda, referida como difusión cultural, subraya la importancia de la transmisión del paquete neolítico como detonante de la expansión del Neolítico y la formación de las primeras culturas neolíticas.

La propuesta de Ammerman y Cavalli-Sforza (1984) es la aproximación más emblemática al problema. Se basa en la adaptación del modelo difusión-reacción de Fisher, aunque incorpora un movimiento de los grupos agrícolas que implementa una presión demográfica constante (crecimiento logístico). Por otro lado, si aceptamos que la expansión del Neolítico se debió a un proceso démico (en desacuerdo Leathwaite 1998; Cruz Berrocal 2012) debemos asumir que este fenómeno tuvo efectos en la variabilidad cultural del primer Neolítico. En este trabajo se plantea testar la hipótesis del autoestop cultural Demic flow raises the possibility that cultural, genetic and linguistic traits with no intrinsic advantage may 'hitchhike'(Ackland et al. 2007: 8714). Dicha hipótesis no difiere mucho del "autoestop genético" de los genetistas (Smith y Haigh 1974), proceso donde la historia evolutiva de un rasgo neutral está sujeto a la evolución de un rasgo ventajoso, sistemáticamente seleccionado. Es decir, cualquier rasgo preexistente, asociado al ventajoso, puede ser transportado junto con la difusión de este con independencia de su valor intrínseco.

Asumiendo dicha hipótesis, elaboramos un modelo computacional para contestar si la expansión del Neolítico en el Mediterráneo occidental pudo ser un proceso de autoestop cultural y si este podría ser documentado en el registro arqueológico $^{6}$. En concreto con este modelo se pretendía investigar si la diversidad cultural neolítica en el Mediterráneo occidental podría estar relacionada con una expansión desde el sur de Italia, explicable por diferentes tipos de autoestop cultural. Nuestro modelo consideró el sistema agrícola/ ganadero como "gen selectivo" y la decoración cerámica como "gen neutro". Para identificar este proceso se adoptó un modelo basado en agentes, la metodología natural para analizar esta clase de problemática.

El modelo de expansión cultural ${ }^{7}$ se diseñó con el software NetLogo (v. 5.05 Wilensky 1999) por su utilidad para explorar la relación entre el comportamiento a escala micro-espacial de los agentes y los resultados a escala macro-espacial que emergen de sus interacciones. Además NetLogo es una plataforma de empleo fácil para construir modelos, realizar simulaciones y visualizarlos, aunque la plataforma ideal no existe (Gilbert y Bankes 2002: 7198). Dicho modelo está basado en el concepto de especificaciones locales (mediante la extensión SIG de NetLogo). Siguiendo nuestro trabajo previo (Bernabeu et al. 2015), a una resolución de $5 \times 5 \mathrm{~km}$, valores entre 1 y 11 de potencialidad de cultivo del trigo son la base para caracterizar el Mediterráneo occidental en combinación con parámetros considerados clave como la pendiente, la temperatura mínima, la media de la temperatura máxima y la media de la precipitación durante el ciclo reproductivo. Junto al parámetro ecológico se tuvieron en cuenta:

a) Movimiento de "expansión" continuada (basado en la expansión de la información económica y cultural): se simuló con dos variantes. La primera de ellas era una analogía del movimiento ola de avance con transmisión a las celdas vecinas siempre y cuando estas sean aptas desde una perspectiva ecológica. La segunda simulaba una expansión a saltos (leap-frog) donde la información se trasmite a cualquier celda apta

\footnotetext{
${ }^{6}$ Pardo Gordó, S. 2015: La diversidad cultural del primer Neolítico en el Mediterráneo occidental. Un análisis desde los sistemas complejos y la simulación basada en agentes. Tesis Doctoral inédita. Universitat de València.

${ }^{7}$ Cultural spread v.1 puede ser descargado desde el repositorio Computational Modeling Library of CoMSES NET (the Network for Computational Modeling in the Social and Ecological Sciences):

http://www.openabm.org/model/4592/version/1/view
} 
para la agricultura en un radio establecido por el investigador (en la interfaz del usuario o por defecto).

b) "Transmisión" de la información: en este caso el modelo plantea tres posibles clases de autoestop cultural: b1) neutral: en la implementación del modelo neutral Kimura 1968) la deriva es el mecanismo que afecta al autoestop cultural; b2) antiguo: aplica un modelo basado en un sesgo temporal (Boyd y Richerson 1985) donde se copia la información cultural de la celda que primero la adoptó; b3) común: aplica un modelo evolutivo basado también en el sesgo donde se adquiere el valor cultural más común del entorno (vecinos).

c) Ratio de "mutación": esta varía entre 0 (ausencia) y 1 (100\% de probabilidad de mutar). En este modelo el investigador establece el parámetro del umbral ecológico (ecological threshold) que define el límite mínimo de exigencia en el cultivo del trigo. Si al iniciar la simulación el valor del parámetro es 6 , solo aquellas parcelas del mundo con un valor igual o superior al umbral se considerarán aptas para el cultivo agrícola. El investigador también controla parámetros como: 1) el punto de origen a partir del que se inicia el proceso de difusión; 2) el tipo de movimiento; 3) la distancia máxima para la elección de la parcela para expandirse; 4) tipo de transmisión de la información (clase de autoestop); 5) tasa de mutación y 6) número de ticks en el que el modelo deja de funcionar. Brevemente, los ticks corresponden al número de veces que el modelo ejecuta los algoritmos programados. Si situamos en 500 el número máximo, el modelo repetirá los procesos diseñados 500 veces y a continuación se detendrá.



Fig. 1. Interfaz del Modelo Basado en Agentes. La estrella marca el punto de origen para la expansión del Neolítico en el Mediterráneo occidental. El color verde indica el valor ecológico para el cultivo del trigo, el color verde más oscuro indica mayor productividad. El color rojo indica que la celda ha recibido agricultura (indica el tiempo, en ticks, de llegada del Neolítico). Las celdas con color gris son aquellas que están por debajo del umbral ecológico considerado. Los botones situados a la izquierda corresponden a los diferentes parámetros de configuración: distancia máxima de la expansión, tipo de movimiento, clase de autoestop cultural, ratio de mutación y otros relacionados con el almacenamiento de los resultados. En color en la versión electrónica.

Trab. Prehist., 74, N. ${ }^{\circ}$ 1, enero-junio 2017, pp. 9-25, ISSN: 0082-5638

doi: $10.3989 /$ tp.2017.12181 
El modelo de expansión cultural también presenta diferentes algoritmos para recopilar los resultados generados in silico según las intenciones del investigador. En nuestro caso, las variables culturales se almacenan en 37 regiones artificiales que agrupan celdas de una malla de $50 \mathrm{x}$ $50 \mathrm{~km}$ según criterios geográficos ${ }^{8}$. Su finalidad era minimizar los problemas de diferente índole asociados al registro arqueológico (p. ej. sesgo, infra/supra representación y naturaleza del depósito arqueológico: excavación y/o prospección). La figura 1 muestra la interfaz del modelo durante los primeros momentos de la simulación, cuando solo ciertas parcelas han recibido el paquete neolítico (economía y cultura). Los parámetros lanzados se muestran a la izquierda.

Finalmente, y como paso previo a la obtención de resultados fiables, se ha validado el modelo para asegurarse que funciona de forma correcta y representa el fenómeno que deseamos investigar. Para comprobar su robustez se han efectuado experimentos para la verificación de la programación, la exploración del número de repeticiones por experimento requerido para lograr resultados estadísticamente representativos, para establecer la relación entre tiempo virtual y tiempo real, para la fijación del valor máximo de ticks por experimento y para la calibración de parámetros (técnica, innovación y umbral ecológico). Sin entrar en los detalles de cada experimento, algo que no pretende este trabajo, sí remarcaremos que los resultados son estadísticamente iguales para 10 repeticiones o 150 . No obstante, por prudencia, hemos repetido cada experimentación 100 veces. Del mismo modo utilizamos los valores 0.004 , $0.008,0.016,0.032$ y 0.064 de ratio de mutación, puesto que los trabajos donde se plantea una tasa de mutación utilizan una ratio con valores cercanos al 0 llegando a sugerir que no supere el 0.1 (Bentley et al. 2011: 540).

\subsection{Resultados in silico y sus implicaciones}

Sin duda la experimentación en laboratorios virtuales mediante la simulación computacional implica que en determinadas ocasiones exista una

${ }^{8}$ Véase n. 7. relación directa (y esperada) entre la modificación sistemática del parámetro y los resultados del modelo. Un ejemplo claro de esta linealidad se observa con la modificación del umbral ecológico: de su incremento se espera la reducción de las parcelas disponible para la expansión de los grupos agrícolas. La figura 2 muestra como el incremento desde 1 a 11 de la variable ecological threshold disminuye el número de celdas ecológicamente aptas para el cultivo del trigo, siendo el coeficiente de correlación entre ambas variables cercano al -0.97 .

Sin embargo la correlación entre la reducción de parcelas y las dinámicas demográficas no está tan clara. Como se observa en la figura 3 el patrón del índice demográfico es independiente del número de parcelas disponibles: un primer momento de crecimiento rápido, entre 20 a 80 ticks, y una posterior estabilización de la curva demográfica a partir de 120 ticks.

No obstante, la modificación sistemática de los parámetros no siempre muestra resultados predecibles. La figura 4 representa la distancia geográfica y cultural de cada una de las regiones consideradas en relación al punto de origen, situado en el sur de Italia, a partir de una expansión basada en ola de avance. En este caso deberíamos esperar que el incremento de la distancia geográfica, calculada con el método Chebyshev (Abello et al. 2002), implique una correlación directa con la distancia cultural, representada con el índice de si-



Fig. 2. Relación entre el límite ecológico considerado en la simulación computacional (X) y el número de celdas disponibles para el cultivo del trigo expresado en $\%$ (Y). El r2 corresponde a la regresión lineal entre ambas variables. 


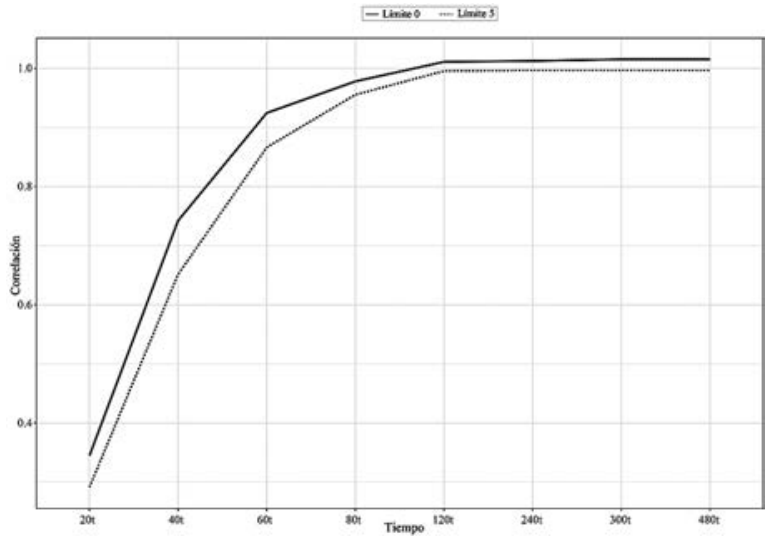

Fig. 3. Comparación del índice demográfico según el límite ecológico utilizado: 0 (línea) 5 (línea y punto). El eje $\mathrm{X}$ representa intervalos de tiempo (ticks) mientras que el eje Y representa el índice demográfico.

militud Brainerd-Robinson (DeBoer et al. 1996), es decir, que a más distancia geográfica haya más distancia cultural. Entonces, ¿cómo debemos interpretar la falta de linealidad? Los arqueólogos suelen atribuirla a la aparición de causas externas (Barton 2014), una interpretación puesta en tela de juicio a partir del análisis desde una perspectiva $C A S$ del registro arqueológico. Desde esta perspectiva los cambios pueden ser 'leídos' como consecuencia de la acumulación de variaciones a pequeña escala que la simulación computacional permite explorar a partir del análisis sistemático de las variables para reducir el número de hipótesis explicativas (Barton 2014). Tal y como observamos, la figura 4 ordena la distancia cultural y geográfica de cada una de las regiones consideradas en relación al sur de Italia siguiendo el criterio de menor a mayor distancia geográfica. En una primera exploración del gráfico vemos que las líneas de tendencias son ascendentes, pero la distancia cultural presenta un patrón basado en dientes de sierra donde en algunas regiones (p. ej. 11 y 17) la distancia cultural es mayor a la esperada y en otras menor (p. ej. 28 y 30). Este ejemplo ilustra perfectamente la obtención de resultados in silico diferentes a los esperados.

¿Pueden ser identificados comportamientos generales distintos si se modifican las reglas de interacción a escala local? El siguiente gráfico presenta esa variabilidad a partir de la modificación sistemática de los parámetros 'distancia' $(1,20$ y

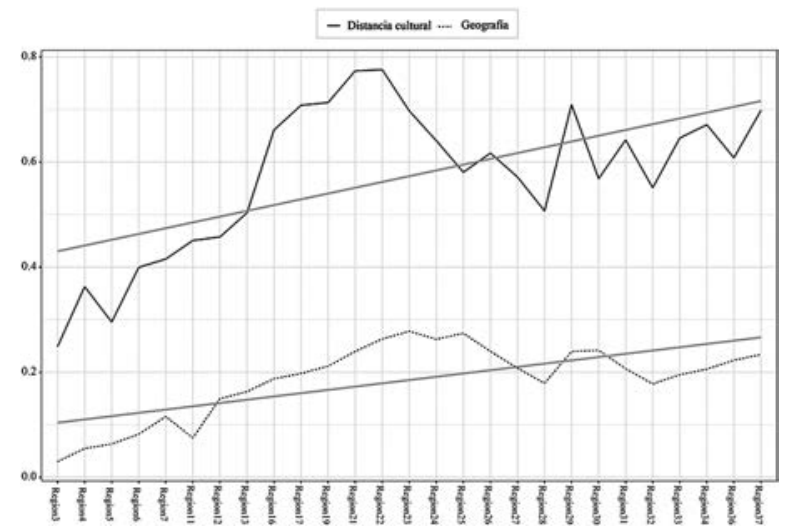

Fig. 4. Comparación entre la distancia geográfica (línea) y la distancia cultural (línea y punto). El eje X representa las regiones virtuales del modelo ordenadas por proximidad geográfica. El eje Y muestra la correlación de las variables consideradas. Las líneas grises indican la tendencia de ambas variables. La parametrización del modelo corresponde a una expansión basada en ola de avance con un umbral ecológico de 5 y una ratio de innovación de 0.004 con un autoestop basado en la deriva.

30) y 'tipo de autoestop cultural' (deriva, copia de la variable cultural de celda más antigua y de la más común). Fuera de las variables mencionadas, los valores del modelo para el límite ecológico (5) y el parámetro innovación $(\mu=0.004)$ son los mismos que en la figura 4. Sin embargo, en la figura 5 se representa la cohesión cultural de todas las regiones mediante el test de Mantel (1967), y la distancia cultural respecto al punto de origen en diferentes momentos temporales. Como se ve, el modelo arroja resultados diferentes según los parámetros modificados: la distancia máxima de expansión y el tipo de autoestop cultural modelado. Por ejemplo, si exploramos la cohesión cultural a lo largo del Mediterráneo occidental (correlación de Mantel) advertimos que los 9 escenarios representados tiene 9 comportamientos diferentes. Este patrón, con alguna matización, muestra también la distancia cultural respecto al punto de origen. En definitiva, el 'modelo de expansión cultural' es un magnífico ejemplo de cómo a partir de las relaciones a escala local de los diferentes agentes (p. ej. reglas de interacción e innovación) emergen fenómenos globales (p. ej. culturas arqueológicas). Los dos casos expuestos (Figs. 4 y 5) nos permiten concluir que el recurso a la simulación computacional genera un abanico diverso de resultados. 


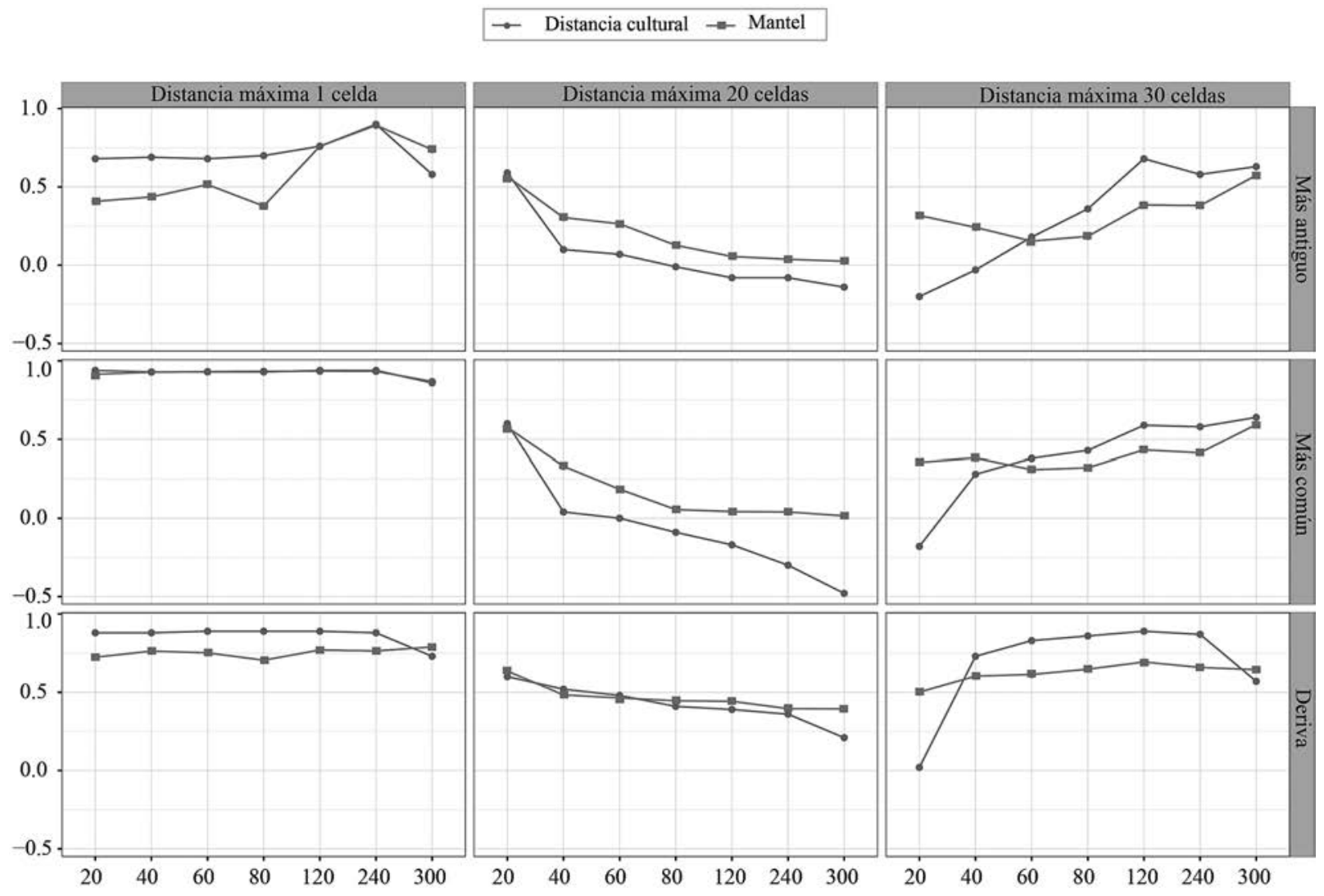

Fig. 5. Resumen de los resultados de la simulación a partir de la modificación sistemática. La distancia explorada ha sido 1, 20 y 30. Los tipos de autoestop cultural considerados son deriva, copia de la variable más común y de la variable más antigua. El límite ecológico considerado es 5 y el valor de $\mu$ es 0.004 . El eje X corresponde a diferentes momentos cronológicos 20 , $40,60,80,120,240,300$ y el eje Y representa la correlación de las variables consideradas: la distancia cultural respecto al punto de origen de la expansión (Brainerd-Robinson, 1952) y la cohesión cultural del Mediterráneo occidental (Mantel 1967).

Esto plantea directamente qué escenario o grupo de ellos se ajusta mejor al registro arqueológico. La selección de los 'indicadores arqueológicos' correctos no es una cuestión baladí ya que, como Shennan y otros (2015) han planteado, diferentes ítems culturales podrían exhibir comportamientos diferentes. Cada variable, en paralelo a su evolución propia, debe ser conocida ya que el recurso de variantes dudosas no parece ser la opción correcta, tal como puede observarse en el trabajo de Parisi y colegas (2008) donde utilizan la lingüística como variable, bajo nuestro de punto de vista incorrecta, para contrastar los resultados simulados. Nosotros planteamos usar como indicador arqueológico la técnica utilizada en la decoración cerámica ${ }^{9}$. No

${ }^{9}$ Veáse n. 6. obstante, tal y como hemos destacado en otra publicación (Bernabeu et al. 2017), la parquedad, la fragmentación y el carácter acumulativo del registro arqueológico debe valorarse siempre cuando se selecciona el indicador arqueológico y, sobre todo, a la hora de compararlo con la información virtual.

La figura 6 ilustra perfectamente esta cuestión a través de una representación filogenética ape package (Paradis et al. 2004) software $\mathrm{R}$ Core Team (2013) mediante un Unweighted Pair Group Method with Arithmetric Mean que asume una velocidad constante de la evolución (Sokal y Michener 1958). La parte superior muestra la información arqueológica disponible en un arco cronológico de 300 años desde la documentación de la primeras producciones cerámicas en las diferentes regiones del Mediterráneo occidental. La 
Representación filogenética datos arqueológicos vs virtuales
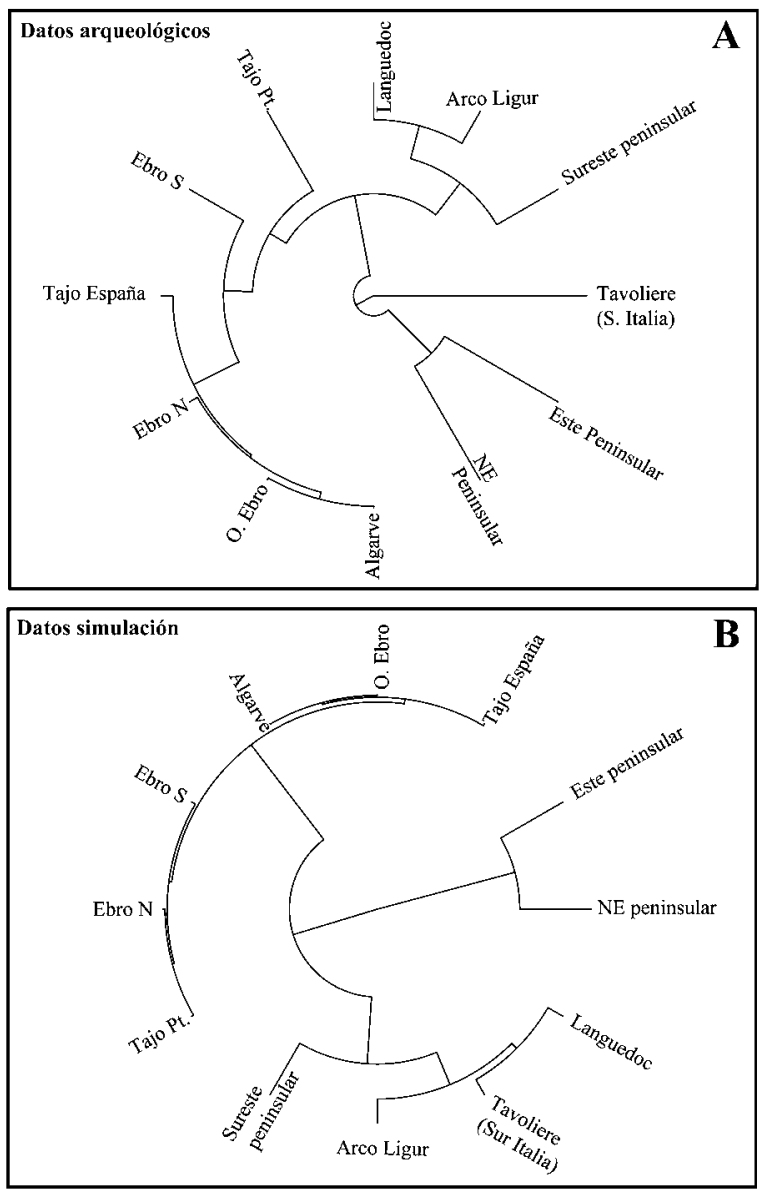

Fig. 6. Ejemplo de representación filogenética de los datos arqueológicos (A) y la información virtual (B) siguiendo un Unweighted Pair Group Method with Arithmetric Mean. Los datos simulados corresponden a los generados de la parametrización del modelo: expansión basada en ola de avance, autoestop cultural basado en la deriva cultural y una ratio de mutación de 0.004 .

parte inferior representa la información cultural disponible resultante de la simulación computacional (Bernabeu et al. 2017) asociada al mismo rango temporal (300 años) utilizando una ratio de mutación de 0.004 , un proceso de autoestop basado en la deriva y una expansión entre celdas vecinas (analogía de la ola de avance).

La figura 6 muestra diferencias notorias entre los resultados arqueológicos (abajo) y aquellos creados in silico (arriba). Su equiparación requiere seleccionar un método de comparación. Los trabajos centrados en el análisis del proceso de expansión per se utilizan la correlación de Pearson entre el tiempo arqueológico (fecha radiocarbónica) y el tiempo virtual (número de ciclos de simulación: ticks o pasos) (p. ej. Bernabeu et al. 2015; Pinhasi et al. 2005). Para la hipótesis del autoestop cultural nos decantamos por el cálculo del índice de correlación de la distancia cultural (CDC). Este índice consiste en una correlación de Pearson a partir de los valores de la distancia cultural obtenida con el coeficiente Brainerd-Robinson de cada una de las regiones virtuales con la correspondiente muestra arqueológica. Por lo tanto, el índice CDC constituye por si mismo una comparación entre el mundo real y el virtual. Además al ser una comparación estadística permite descartar los escenarios virtuales sin significación estadística (pvalue $>0.05$ ), es decir, los escenarios que no se ajustan a la premisa de inicio.

La aplicación del modelo $A B M$ en relación a la expansión del Neolítico en el Mediterráneo occidental sugiere: a) que, durante dicho proceso, el autoestop cultural influyó en la variabilidad de las primeras producciones cerámicas, sobre todo, en las regiones asociadas a la cultura impresa/ cardial y b) que la diversidad cultural puede ser explicada utilizando métodos y técnicas propias de las ciencias biológicas.

\section{ALGUNAS REFLEXIONES FINALES}

En el presente trabajo se ha argumentado la necesidad de analizar el registro arqueológico desde una perspectiva $C A S$ que destierra del pensamiento arqueológico la correlación entre complejidad social y sociedades más complejas. Solo un análisis $C A S$ permite comprender que el comportamiento y evolución de las sociedades humanas se plasma en diferentes cuestiones, como la diversidad cultural y no se limita a las relacionadas con la organización social.

Del mismo modo, la simulación computacional se está convirtiendo en un incipiente método para el análisis del pasado (Lake 2015) pero más en los sistemas universitarios anglosajones que en la arqueología peninsular donde su visibilidad aún es escasa. Queremos destacar que la aplicación de la simulación computacional en nuestra disciplina no busca reconstruir el pasado de forma fidedigna, sino generar historias alternativas sobre la forma- 
ción del registro arqueológico. Dado el carácter fragmentario y escaso de los restos arqueológicos no caben inferencias sobre el comportamiento de los grupos humanos del pasado (Barton 2014) sin recurrir al laboratorio virtual. No obstante, la aceptación de la simulación computacional no implica un uso a ciegas de los modelos computacionales, cuya utilidad reside en que sean interpretables de forma correcta, y sobre todo, coherente. Por ello abogamos por recurrir a la modelización siempre y cuando haya un trabajo previo/posterior centrado en la comprensión del registro arqueológico. En este sentido, algunos trabajos son excelentes desde una perspectiva de método pero carecen de una interpretación correcta de los resultados computacionales. La investigación de Banks y otros (2013) sobre la expansión de las grandes culturas neolíticas en Europa a partir de la modelización del nicho ecológico es un buen ejemplo del riesgo de caer en una interpretación errónea de los resultados. En dicho trabajo, asumiendo falta de información arqueológica, se predice la existencia de áreas como el interior de la península ibérica y el Norte de África relacionadas con el nicho ecológico cardial pero sin este tipo cerámico (Banks et al. 2013: 2751). El supuesto de que no hay información arqueológica es erróneo: se conocen yacimientos relacionados con la cultura cardial en ambas áreas (Gilman 1975; Jiménez Guijarro 2008) y, además, absence of evidence is not evidence of absense (Kuhn y Steiner 2006: 957).

En este trabajo se ha demostrado que los modelos evolutivos (una analogía del autoestop genético) tomados prestados de la Biología son una herramienta muy útil para el análisis del registro arqueológico. Otro estudio previo (Crema et al. 2014a) dejó claro que, además, sus métodos son realmente útiles para el análisis del registro. Sin embargo la aplicación de los modelos biológicos globales no están exentos de críticas debido al carácter particularista de las interacciones humanas (p. ej. Sahlins 1998). Esta contraposición entre modelos biológicos y modelos antropológicos ha promovido un amplio debate en torno a las diferencias entre la genética y la conducta (véase VanPool 2008 para detalles). Nosotros, aunque abogamos por la complementariedad de ambos, nos decantamos por el uso de los modelos biológicos: a) los postulados evolutivos permiten establecer reglas simples de interacción y habilidades de los agentes (Miller y Page 2007: 180) para modelar su evolución; b) como el registro arqueológico es una consecuencia de las interacciones entre agentes evolutivos (p. ej. individuos, grupos y/o sociedades) su estudio debe abordarse desde una perspectiva teórico-metodológica evolucionista donde los modelos biológicos son el enfoque natural.

Finalmente la utilización del llamado "computerrarium" (Epstein y Axtell 1997) implica la emergencia de nuevos retos en la Arqueología como la selección de la información oportuna (proxy). El registro ofrece una gran variedad de información arqueológica que puede y, sobre todo, debe ser utilizada para testar y ajustar los modelos arqueomáticos. En un trabajo reciente en torno a la expansión del Neolítico en la península ibérica hemos demostrado la necesidad de auditar la muestra ya que una utilización indiscriminada de la información arqueológica puede arrojar interpretaciones erróneas (Bernabeu et al. 2015; Pardo Gordó et al. 2015).

En definitiva, la aplicación de la simulación computacional puede ayudarnos a comprender mejor las sociedades humanas del pasado al permitir estudiar fenómenos como las dispersiones humanas o la evolución cultural bajo condiciones controladas por el investigador.

\section{AGRADECIMIENTOS}

A los Dres. Joan Bernabeu Aubán (Universitat de València) y C. Michael Barton (Arizona State University), codirectores de la Tesis Doctoral de la que deriva este trabajo. A los dos revisores/as anónimos/as puesto que sus comentarios han incrementado notablemente la calidad de este trabajo. Finalmente, el autor agradece al Centre de Càlcul de la Universitat de València el apoyo técnico y permiso para utilizar el nodo local MULTIVAC de la red de supercomputación española para llevar a cabo las simulaciones.

\section{BIBLIOGRAFÍA}

Abello, J. M.; Pardalos, P. M. y Resendre, M. G. C. 2002: Handbook of Massive Data Sets. Springer Science. Nueva York.

Ackland, G. J.; Signitzer, M.; Stratford, K. y Cohen, M. H. 2007: "Cultural hitchhiking on the wave of 
advance of beneficial technologies". Proceedings of the NationalAcademy of Sciences of the United States of America 104: 8714-8719.

Aldenderfer, M. 1998: "Quantitative methods in archaeology: a review of recent trends and developments". Journal of Archaeological Research 6: 91-120.

Allen, P. R. 1997: Cities and regions as self-organizing systems. Gordon and Breach. Londres.

Ammerman, A. J. y Cavalli-Sforza, L. L. 1971: "Measuring the Rate of Spread of Early Farming in Europe". Man 6: 674-688.

Ammerman, A. J. y Cavalli-Sforza, L. L. 1973: “A population model for the diffusion of early farming in Europe". En C. Renfrew (ed.): The explanation of culture change:models in Prehistory. Duckworth. Londres: 343-358.

Ammerman, A. J. y Cavalli-Sforza, L. L. 1979: “The Wave of Advance Model for the spread of agriculture in Europe". En K. L. Cooke y C. Renfrew (eds.): Transformations: mathematical approaches to culture change. Academic Press. Nueva York: 275-294.

Ammerman, A. J. y Cavalli-Sforza, L. L. 1984: The neolithic transition and the Genetics of population in Europe. Princeton University Press. PrincetonNueva Jersey.

Aoki, K.; Shida, M. y Shigesada, N. 1996: "Travelling Wave Solutions for the Spread of farmers into a region occupied by Hunter-Gatherers". Theoretical population biology 50: 1-17.

Banks, W.; Antunes, N.; Rigaud, S. y d'Errico, F. 2013: "Ecological constraints of the first prehistoric farmers in Europe". Journal of Archaeological Science 40: 2746-2753.

Barabási, A.-L. 2005: "Network Theory. The emergence of the creative enterprise". Science 308: 639-641.

Barabási, A.-L. 2012: "The network takeover". Nature Physics 8: 14-16.

Barton, C. M. 2013: "Stories of the past or science of the future? archaeology and computational social science". En A. Bevan, y M. Lake (eds.): Computational approaches to archaeological spaces. Left Coast Press. Walnut Creek: 151-178.

Barton, C. M. 2014: "Complexity, social complexity, and modeling". Journal of Archaeological Method and Theory 21, 2: 306-324 doi:10.1007/s10816-0139187-2.

Barton, C. M.; Ullah, I. I. y Bergin, S. 2010: "Land use, water and Mediterranean landscapes: modelling long-term dynamics of complex socio-ecological systems". Philosophical Transactions of the Royal Society A: Mathematical, Physical and Engineering Sciences 368: 5275-5297.

Barton, C. M.; Ullah, I. I. T.; Bergin, S. M.; Mitasova, H. y Sarjoughian, H. 2012: "Looking for the future in the past: long-term change in socioecological systems". Ecological Modelling 241: 42-53.
Bell, J. 1987: "Simulation modeling in archaeology: Reflections and trends". European Journal of Operational Research 30: 243-245.

Bentley, R. A. 2003: "An introduction to Complex Systems". En R. A. Bentley y H. D. G. Maschner (eds.): Complex systems and archaeology: empirical and theoretical applications. Foundations of Archaeological Inquiry, University of Utah Press. Salt Lake City: 9-23.

Bentley, R. A. y Maschner, H. D. G. 2003: Complex systems and Archaeology: empirical and theoretical applications. Foundations of Archaeological Inquiry, University of Utah Press. Salt Lake City.

Bentley, R. A. y Maschner, H. D. G. 2008: "Complexity theory". En R. A. Bentley, H. D. G. Maschner y C. Chippindale (eds.): Handbook of Archaeological Theories. Altamira Press. Lanham: 245-270.

Bentley, R.A.; Ormerod, P. y Batty, M. 2011: "Evolving Social Influence in Large Population". Behavioral Ecology and Sociobiology 65(3): 537-546.

Bernabeu J. 2016: "Dispersiones humanas y culturales durante la Transición Neolítica: Sistemas Complejos y Prehistoria". En Del neolític a l'edat del bronze en el Mediterrani occidental. Estudis en homenatge a Bernat Martí Oliver. Trabajos Varios Servicio Investigación Prehistórica 119. Valencia: 51-60.

Bernabeu, J.; Barton, C. M.; Pardo Gordó, S. y Bergin, S. M. 2015: "Modeling initial Neolithic Dispersal. The first agricultural groups on West Mediterranean". Ecological Modeling 307: 22-31.

Bernabeu, J.; Moreno, A. y Barton, C. M. 2013: “Complex systems, social networks, and the evolution of social complexity in the East of Spain from the Neolithic to Pre-Roman iimes". En M. Cruz Berrocal, L. García Sanjuán y A. Gilman Guillén (eds.): The Prehistory of Iberia: debating early social stratification and the state. Routledge. New York: 53-73.

Bernabeu, J.; Manen, C. y Pardo-Gordó, S. 2017: "Spatial and temporal diversity during the Neolithic spread in the Western Mediterranean. The first pottery production”. En O. García Puchol y D. C. Salazar-García (eds.): Times of Neolithic transition along the western Mediterranean. Fundamental Iusses in Archaeology, Springer, Cham. 10.1007/9783-319-52939-4.

Bertalanffy, L. von 1951: "General System Theory, a new approach to unity of science". Human Biology 23: 303-361.

Binford, L. 1962: "Archaeology as anthropology". American Antiquity 28: 217-225.

Bonabeau, E. 2002: "Agent-based modeling: methods and techniques for simulating human systems". Proceedings of the National Academy of Sciences 99: 7280-7287.

Boyd, R. y Richerson, P. J. 1985: Culture and the Evolutionary Process. University of Chicago Press. Chicago. 
Robinson, WS.; Brainerd G. 1952: Robinson's Coefficient of Agreement-A Rejoinder. American Antiquity 18 (1): 60-61.

Brughmans, T. 2013: "Thinking through networks: a review of formal network methods in archaeology". Journal of Archaeological Method and Theory 20: 623-662.

Chapman, Robert. 2003. Archaeologies of complexity. Londres: Routledge.

Childe, G. V. 1925: The dawn of European civilisation. Kegan Paul. London.

Cilliers, P. 1998: Complexity and Postmodernism: understanding complex systems. Routledge. London.

Cohen, M. H. 1992: "Nonlinearity, disorder, the spread of the Neolithic farming and the origin of the IndoEuropean languages". En F. Abdullaev, A.R. Bishop y S. Pnevmatikos (eds.): Nonlinearity with disorder. Proceedings in Physics. Springer. Berlin: 161-170.

Collar, A.; Coward, F.; Brughmans, T. y Mills, B. J. 2015: "Networks in archaeology: phenomena, abstraction, representation". Journal of Archaeological Method and Theory 22: 1-32.

Conolly, J.; Colledge, S. y Shennan, S. 2008: "Founder effect, drift, and adaptive Change in domestic crop use in early Neolithic Europe". Journal of Archaeological Science 35: 2797-2804.

Cooke, K. L.y Renfrew, C. 1979: "An experiment on the simulation of culture change". En K. L. Cooke y C. Renfrew (eds.): Transformations: mathematical approaches to culture change. Academic Press. Nueva York: 327-348.

Costopoulos, A. 2010: "For a theory of archaeological simulation". En A. Costopoulos y M. Lake (eds.): Simulating change: Archaeology into the twentyfirst century. Foundations of Archaeological Inquiry, University of Utah Press. Salt Lake City: 21-27.

Costopoulos, A. y Lake, M. 2010: Simulating change: Archaeology into the twenty-first century. Foundations of Archaeological Inquiry. University of Utah Press Salt Lake City.

Costopoulos, A., Lake, M. y Gupta, N. 2010.: "Introduction". En A. Costopoulos y M. Lake (eds.): Simulating change: Archaeology into the twentyfirst century. Foundations of Archaeological Inquiry, University of Utah Press. Salt Lake City: 1-8.

Coward, F. 2013: "Grounding the net: social networks, material cultural and geography in the epipaleolithic and early neolithic of the Near East (21.000-6000 cal. BCE)". En C. Knappet (ed.): Network analysis in Archaeology: new regional approaches to interaction. Oxford University Press. Oxford: 247-280.

Crema, E. R.; Kerig, T. y Shennan, S. 2014a: "Culture, space, and metapopulation: a simulation-based study for evaluating signals of blending and branching". Journal of Archaeological Science 43: 289-298.

Crema, E. R.; Edinborough, K.; Kerig, T. y Shennan, S. 2014b: "An approximate bayesian computation approach for inferring patterns of cultural evolu- tionary change". Journal of Archaeological Science 50: $160-170$.

Cruz Berrocal, M. 2012: "The Early Neolithic in the Iberian Peninsula and the Western Mediterranean: a review of the evidence on migration". Journal of World Prehistory 25(3-4): 123-156.

DeBoer, W. R.; Kintigh, K. y Rostoker, A. G. 1996: "Ceramic seriation and site reoccupation in Lowland South America". Latin American Antiquity 7: 263278.

Doran, J.E. 1970: "Systems theory, computer simulations, and archaeology". World Archaeology 1: 289-298.

Doran, J. E. y Hodson, F. R. 1975: Mathematics and computer in Archaeology. Edinburgh University Press. Edinburgh.

Doran, J. E.; Palmer, M.; Gilbert, N. G. y Mellars, P. 1994: "The EOS project: modeling Upper Palaeolithic social change". En N. G. Gilbert y J. E. Doran (eds.): Simulating societies: the computer simulation of social phenomena. UCL Press. London: 195-222.

Edinborough, K.; Shennan, S.; Crema, E. R. y Kerig, T. 2015: "An ABC of lithic arrowheads: a case study from south-eastern France". En K. Brink, S. Hydén, K. Jennbert, L. Larsson y D. Olausson (eds.): Neolithic diversities: perspectives From a conference in Lund, Sweden. Department of Archaeology and Ancient History, Lund University. Lund: 213-223.

Epstein, J. 1999: "Agent-Based Computational Models and Generative Social Science". Complexity 4, 41-60.

Epstein, J. y Axtell, R. 1997: "Artificial societies and generative social science". Artificial Life and Robotics 1: 33-34.

Farkas, I.; Helbing, D. y Vicsek, T. 2003: "Human waves in stadiums". Physica A: statistical mechanics and its applications 330: 18-24.

Feigenbaum, M. J. 1980: Universal Behavior in Nonlinear Systems. Los Alamos. Science: 4-27.

Fernandez Uriel, P. 1995: "Algunas precisiones sobre el sistema fiscal romano". Espacio Tiempo y Forma. Serie II, Historia Antigua 8: 159-182.

Fort, J. y Méndez, V. 1999a: "Time-delayed theory of the Neolithic transition in Europe". Physical Review Letters 82: 867-870.

Fort, J. y Méndez, V. 1999b: "Reaction diffusion waves of advance in the transition to agricultural economics". Physical Review E 60: 5894-5901.

García-Valdecasas, J. I. 2011: "La simulación basada en agentes: una nueva forma de explorar fenómenos sociales". Reis 136: 91-110.

Gell-Mann, M. 1994: The quark and the jaguar: adventures in the simple and the complex. W. H. Freeman, London

Gell-Mann, M. 1999: “Complex adaptive systems”. En G. Cowan, D. Pines y D. Meltzer (eds.): Complexity: metaphors, models, and reality. Addison-Wesley Publishing. Reading-Massachusetts:17-45. 
Gilbert, N. y Bankes, S. 2002: "Platforms and methods for agent-based modeling". Proceedings of the $\mathrm{Na}$ tional Academy of Sciences of the United States of America 99: 7197-7198.

Gilman, A. 1975: The later prehistory of Tangier (Morocco). Bulletin of the American School of Prehistoric Research 29, Peabody Museum, Harvard University. Mass.

Gilman, A. 2013: "Were there states during the Later Prehistory of Southern Iberia". En M. Cruz Berrocal, L. García Sanjuán y A. Gilman Guillén (eds.): The Prehistory of Iberia: debating early social stratification and the state. Routledge. New York: 10-28.

Helbing, D. 2010: "Pluralistic modeling of complex system". Science and Culture 76: 315-329.

Helmreich, S. 1998: Silicon second nature: culturing artificial life in a digital world. University of California Press, Oakland, California.

Helmreich, S. 1999: 'Digitizing 'Development'. Baline water temples, complexity and the politics of simulation". Critique of Anthropology 19(3): 249-265

Holland, J. 1975: Adaptation in natural and artificial systems. MIT Press, Boston.

Holland, J. 1995: Hidden order: how adaptation builds complexity. Addison-Wesley. Redwood City.

Izquierdo, L.; Galán, J. M.; Santos, J. I. y Olmo, R. del 2008: "Modelado de sistemas complejos mediante simulación basada en agentes y mediante dinámica de sistemas". EMPIRIA. Revista de Metodología de Ciencias Sociales 16: 85-112.

Jiménez Guijarro, J. 2008: "El horizonte cardial del interior de la Península Ibérica". En M. Hernández, J. Soler y J. A. López Padilla (eds.): IV congreso del Neolítico peninsular (Alicante 2006) 2: 348-355. Alicante.

Johnson, A.W. y Earle, T. K. 2000: The evolution of human societies: from foraging group to agrarian state. Stanford University Press. Stanford.

Johnson, G.A. 1982: "Organizational structure and scalar stress". En C. Renfrew, M. Rowlands y B. A Segraves-Whallon (eds.): Theory and Explanation in Archaeology. Academic Press. Southampton: 389-421.

Khalil, E. 1995: "Nonlinear thermodynamics and social science modelling: fad cycles, cultural development and identificational slips". American Journal of Economics and Sociology 54(4) 423-438.

Kimura, M. 1968: "Evolutionary Rate at the Molecular Level". Nature 217: 624-626.

Kohler, T. A. 1993: "News from the North American Southwest: Prehistory on the Edge of Chaos". Journal of Archaeological Research 1, 267-321.

Kohler, T. A. 2000: "Putting social sciences together again: An introduction to the volume". En T. A. Kohler y G. J. Gumerman (eds.): Dynamics in human and primate societies: agent-based modelling of social and spatial processes. Oxford University Press. Nueva York: 1-20.
Kohler, T. A. 2012: “Complex systems and archaeology". Archaeological theory today 93-123.

Kohler, T. A. y Gumerman, G. J. 2000: Dynamics in human and primate societies: agent based modeling of social and spatial processes. Oxford University Press, Nueva York.

Kuhn, S. y Steiner, M. 2006: "What's a Mother to Do?" Current Anthropology 47(6), 953-980.

Lake, M. 2001: "Numerical modelling in Archaeology". En D. R. Brothwell y A. M. Pollard (eds.): Handbook of Archaeological Sciences. John Wiley \& Sons. Chichester: 723-732.

Lake, M. 2013: "Trends in archaeological simulation." Journal Archaeological Method and Theory. 1-30. doi:10.1007/s10816-013-9188-1

Lake, M. 2015: "Explaining the past with ABM: on modelling philosophy". En G. Wurzer, K. Kowarik y H. Reschreiter (eds.). Agent-based modeling and simulation in archaeology. Advances in Geographic Information Science, Springer. Berlin: 3-35.

Leathwaite, J. 1988: "The transition to food production: a Mediterranean perspective". En M. Zvelebil (ed.): Hunters in transition. Mesolithic societies of temperate Eurasia and their transition to farming. Cambrigde University Press. Londres: 53-66.

Lorenz, E. N. 1995: The essence of chaos. University of Washington Press.

Lull, V. 1983: La Cultura de El Argar: un modelo para el estudio de las formaciones económico-sociales prehistóricas. Akal. Madrid.

Mantel, N. 1967: "The detection of disease clustering and a generalized regression approach". Cancer Research 27: 209-220.

McGlade, J. 2005: "Systems and simulacra: modeling, simulation, and archaeological interpretation". En H. D. G Maschner y C. Chippindale (eds.): Handbook of Archaeological Methods. Altamira Press. Oxford: 554-602.

McGlade, J. y van der Leeuw, S. 1997: "Introduction: archaeology and non-linear dynamics -new approaches to long-term change". En S. van der Leeuw y J. McGlade (eds.): Time, process and structured transformation in Archaeology. Routledge. London: $1-31$.

Miller, J. H. y Page, S. E. 2004: “The standing ovation problem". Complexity 9: 8-16.

Miller, J. H. y Page, S. E. 2007: Complex adaptive systems: an introduction to computational models of social life. Princeton University Press. PrincetonNew Jersey.

Mitchell, M. 2009: Complexity: a guided tour. Oxford University Press. Oxford.

Mithen, S. 1990: Thougtful foragers: a study of prehistoric decision makers. University Press. Cambridge.

Mithen, S. 1994: "Simulating prehistoric hunter-gatherer". En N. G. Gilbert y J. E. Doran 8eds.): Simulating societies: the computer simulation of social phenomena. UCL Press. London.

Trab. Prehist., 74, N. ${ }^{\circ}$ 1, enero-junio 2017, pp. 9-25, ISSN: 0082-5638

doi: $10.3989 /$ tp.2017.12181 
Ortman, S. G.; Varien, M. D. y Gripp, T. L. 2007: “Empirical bayesian methods for archaeological survey data: an application from the Mesa Verde Region". American Antiquity 72: 241-272.

Paradis, E.; Claude, J. y Strimmer, K. 2004: “APE: analyses of phylogenetics and evolution in R language". Bioinformatics 20: 289-290.

Pardo Gordó, S.; Bernabeu Aubán, J.; García Puchol, O.; Barton, C. M. y Bergin, S. M. 2015: "The origins of agriculture in Iberia: a computational model". Documenta Praehistorica XLII: 117-131.

Parisi, D.; Antinucci, F.; Natale, F. y Cecconi, F. 2008: "Simulating the expansion of farming and the differentation of European languages". En B. Laks (ed.): Origin and evolution of languages: approaches, models, paradigms. Equinox Publishing. Londres: 192-214.

Parunak, H. V. D.; Savit, R. y Riolo, R. L. 1998: "Agent-based modeling vs equation-based modeling: a case study and user's guide". En J. S. Sichman, R. Conte y N. G. Gilbert (eds.): Multiagent systems and agent-based simulation. Lecture notes in artificial inteligence. Springer. Berlin: 10-25.

Pinhasi, R., Fort, J. y Ammerman, A. J. 2005: "Tracing the origin and spread of agriculture in Europe". PLoS Biol 3: e410.

Premo, L. 2010: "Equifinality and explanation: the role of agent-based modeling in postpositivist archaeology". En A. Costopoulous y M. Lake (eds.): Simulating change:Archaeology into the twenty-first century.University of Utah Press. Salt Lake City: 28-37.

Prigogine, I., 1987: "Exploring complexity". European Journal of Operational Research 30: 97-103.

Quesada, M. y Hassan, S. 2012: "La investigación mediante simulación social multiagente". En M. Arroyo e I. Sábada (ed.): Metodología de la investigación social. Técnicas innovadoras y sus aplicaciones. Síntesis. Madrid: 321-344

R Core Team 2013: R: A language and environment for statistical computing. R Foundation for Statistical Computing. Viena, Austria.

Railsback, S. F. y Grimm, V. 2012: Agent-based and Individual-based Modeling: A Practical Introduction. Princeton University Press. New Jersey.

Rubio-Campillo, X. 2016: "Model selection in historical research using approximate bayesian computation". PLoS ONE 11(1): e0146491. doi:10.1371/ journal.pone.0146491
Sahlins, M. 1998: Uso y abuso de la Biología. Una crítica antropológica de la sociobiología. Siglo XXI España. Madrid.

Shennan, S. 2002: Genes, memes and human history. Darwinian archaeology and cultural evolution. Thames \& Hudson. London.

Shennan, S.; Crema E.y Kerig, T. 2015: "Isolationby-distance, homophily, and 'core' vs. 'package' cultural evolution models in Neolithic Europe". Evolution and Human Behavior 36(2): 103-109.

Silva, F. y Steele, J. 2014: "New methods for reconstructing geographical effects on dispersal rates and routes form large-scale radiocarbon databases". Journal of Archaeological Science 52: 609-620.

Sinclair, T. R. y Seligman, N. G. 1996: "Crop modeling: from infancy to maturity". Agronomy Journal 88: 698-704.

Smith, J. M. y Haigh, J. 1974: "The hitch-hiking effect of a favourable gene". Genetics Research 23: 23-35.

Sokal, R. y Michener, C. 1958: "A statistical method for evaluating systematic relationships". Science Bulletin 38: 1409-1438.

Steele, J. 2009: "Human dispersals: mathematical models and the archaeological record". Human Biology 81: 121-140.

Strogatz, S., 1994: Nonlinear dynamics and chaos. Perseus Books. Reading.

Van der Leeuw, S. y McGlade, J. 1997: Time, process and structured transformation in archaeology. Routledge, London.

VanPool, C. S. 2008: "Agents and cultural transmission”. En M. J. O’Brien (ed.): Cultural transmission and archaeology. Issues and case studies. Society for American Archaeology. Washington: 190-200.

Waldrop, M. M. 1992: Complexity: the emerging science at the egde of order and chaos. Simon and Schuster. Nueva York.

Wilensky, U. 1999: NetLogo. http://ccl.northwestern. edu/netlogo

Wobst, H. M. 1974. "Boundary conditions for Paleolithic social system: a simulation approach". American Antiquity 39: 147-178.

Wright, H. T. y Zeder, M. 1977: "The simulation of a linear exchange system under equilibrium conditions". En T. K. Earle y J. E Ericson (eds.): Exchange systems in Prehistory. Academic Press. Nueva York: 233-352.

Yoffee, N. 2005: Myths of the archaic state: Evolution of the earliest cities, states, and civilizations. Cambridge University Press. Nueva York. 